\title{
Las complicaciones varicosas en la embarazada
}

\author{
Doctores Conzalo Echeverri y Humberto Correa.
}

Preocupados por el problema que en algunas ocasiones se piantea con las compiicaciones venosas de orden varicoso en las embarazadas, hemos querido traer este tema para su análisis y discusión en el seno de la Sociedad Colombiana de Obstetricia y Ginecología.

Aunque podría considerarse como un asunto más bien trivial en el cual el obstetra no tiene ni debe hacer más que limitarse a prescribir el uso de vendajes y medias elásticas, y a consolar a su raciente diciéndole que no hay qué hacer, o que se aplazará ia solución del caso hasta que se termine el estado puerperal, es bien cierto que hay algunas ocasiones en que la enfermedad varicosa acarrea tal cúmulo y calidad de síntomas y trastornos a la embarazada, que el obstetra no puede eludir el compromiso de tratar, por lo menos, de dar un alivio, si no una solución radical.

Es de todos conocida la exacerbación y desarrollo que durante el embarazo adquieren las várices. Favorecidas por un factor constitucional y de tonicidad tisular peculiar a cada organismo, que estamos obligados a aceptar y que da por resultado la debilidad de la pared venosa, las venas se van dilatando, sea por exceso de presión desde ia luz venosa hacia afuera, sea por falta de soporte de los tejidos que rodean la vena. Ahora bien, el embarazo exagera la presión endovenosa en los miembros inferiores por varias causas: Hidremia o sea aumento dei volumen circulante, y compresión por el útero grávido de los grandes troncos pélvicos. Si a esto se agrega el reblandecimiento general de todos los tejicos del organismo, que inciuiría la pared venosa misma y la fascia y tejido areolar que rodean a las venas, se comprenderá fácilmente que el estado grávido reúne todas las condiciones para hacer que las venas sufran dilataciones varicosas. 
Los cambios patológicos esenciales consisten en: a) Dilatación; b) Elongación y tortuosidad; c) Pérdida de elasticidad con aumento del tejido conectivo fibroso; d) Variaciones en el espesor de la pared, y e) Atrofia o desaparición compieta de las válvulas. Estos cambios se hacen de preferencia sobre el sistema de las safenas, pero puede presentarse la insuficiencia de las venas comunicantes acarreando estasis y en algunos casos inversión del sentido de la corriente sanguínea.

El caso de ocurrencia corriente, que podriamos estimar en más de un $50 \%$ de las embarazadas, conssite en dilataciones varicosas de la pierna, más o menos pronunciadas, que se acompañan de zonas telangiectásicas de los muslos y de un notorio aumento de ia fragilidad capilar que da como resultado la frecuente aparición de pequeñas equimosis ne los miembros inferiores. Estos estados varicosos son con mucha frecuencia asintomáticos, o pueden presentar en casos más avanzados discreto edema maleolar y alguna sensación dolorosa no permanente sino ocacionada por el ejercicio o la estación de piés prolongada. Este dolor ocurre generalmente a nivel de las dilataciones varicosas mismas, sin envolver en los sintomas a todo el miembro inferior.

Son estos los casos en que no hay objeto en considerar una terapéutica activa, y la sola prescripción de un poco de reposo, o eventualmente el uso de medias o vendajes elásticos, pueden resolver el problema. Una vez terminado el embarazo desaparecen las dilataciones venosas sin dejar rastros, hasta un nuevo embarazo en que son en general un poco más avanzados.

Pero si este grupo de pacientes no tienen problema con sus várices ni se lo ocasionan al obstetra, hay otro que es el que ha motivado esta ponencia, y en ei cual la situación es bien diferente tanto para la paciente como para el médico.

Se trata de ios casos de grandes racimos varicosos que se extienden a todo lo largo de la pierna y del muslo, saliéndose del territorio de las safenas para abarcar todas las venas superficiales, y de entre éstas muy especialmente las lei tercio superior de la cara posterior del muslo. Estos casos se complican a veces con la aparición de grandes paquetes venosos de los grandes labios y de las mismas paredes vaginales.

En este tipo de pacientes unas veces los síntomas no pasan de un poco de dolor, edema y sensación de pesantez, pero hay otros casos, por fortuna no muy frecuentes, en que el doior se' generaliza en tolo el miembro inferior, dificultando y en ocasiones 
haciendo imposible la marcha. Hay considerable edema y calambres musculares de las piernas que ocasionan dolores intensos. También se presentan fenómenos de periflebitis que hacen todavís más doloroso el cuadro clínico. Pueden ocurrir, como an cualquier paciente varicoso, rupturas venosas o accidentes de tromboflebitis, que deben considerarse como complicaciones de carácter urgente y ser tratados en conseculencia.

Estos son los casos que consideramos que deben requerir una conducta activa, pues las pacientes necesitan alivio para sus dolores y malestares, y deben ponerse a cubierto de complicaciones graves. ¿Cuál sería esta conducta? Nuestra experiencia personal es muy reducida en este campo, y por eso nos limitaremos a pasar una revista resumida de los principaies sistemas puestos en práctica.

El sistema clásico de las inyecciones esclerosantes en el tratamiento de las várices de las embarazadas ha sido discutido. Existe en general el concepto de que este tipo de tratamiento está contraindicado en el embarazo, por la presunta lesión renal que las sustancias inyectadas producirán. Sinembargo, parece que en reailidad la embarazada puede tratarse por este sistema en las mismas condiciones que cualquier paciente varicoso. Al respecto anota Greenhill que "no hay ningún peligro en el tratamiento de las venas varicosas en el embarazo, y a pesar de que hay una gran incidencia de recurrencias, el tratamiento se justifica".

La serie mayor de casos que hemos encontrado, tratados por este sistema, la presenta Edward Solomons sobre 600 pacientes con todos los tipos de várices desde la vulva hasta la pierna. Ei único obstáculo para el tratamiento consistía en la historia de trombosis profunda, caso en el cual no se inyectaban. Los agentes usados fueron quinina uretano (50 casos), soluciones jabonosas (248 casos) y sotradecol, o sea tetradecyl sulfato sódico (202 casos). Solomons sostiene que las críticas convencionaies al tratamiento por inyección no son justas; que la inyección detiene la degeneración progresiva, trae un rápido alivio de los síntomas y con frecuencia una curación permanente. En su serie de casos el alivio temporal fue notable y se puede considerar constante en todas las pacientes. Se presentaron recurrencias tardias entre el 50 y 80 por ciento de los casos; pero anota que a pesar del aito índice de recurrencias hay curaciones permanentes suficientes para justificar un tratamiento tan sencillo. No hubo complicaciones de carácter obstétrico ni puerperal, y solamente en pocos casos tuvo flebitis químicas transitorias. 
En cuanto al tratamiento quirúrgico, está demostrado también que es practicable durante el embarazo, sin que acarree consecuencias nocivas. Se han utilizado las diferentes técnicas de fleboextracción y ligaduras múitiples, combinadas o no con sustancias esclerosantes, y existe abundante literatura al respecto. Se puede concluir de estas publicaciones que no hay consecuencias especiales en cuanto al embarazo, parto o puerperio, y que en general los síntomas han cedido en forma casi inmediata, presen tándose eso sí buen número de recidivas. Hay que tener en cuenta que cuando se trata de lesiones varicosas muy extensas, que se salen del territorio de las safenas, la operación debe hacerse bastante ampiia y aun asi muchas veces quedan territorios varicosos sin intervenir, que siguen ocasionando s'ntomas molestos. Iguaimente se anota que a pesar de pruebas correctas preoperatorias de Trendelemburg y de Perthes, quedan con frecuencia edemas tibiales y maleolares de duración prolongada. Queda también el problema del labiocele o várices vulvares, que de tratarse quirúrgicamente requeriran ligaduras in situ, lo cuai es un procedimiento arriesgado por la gran fragilidad de las paredes venosas en este sitio, la dificultad de la disección correcta y el riesgo inmediato de rupturas venosas con la consiguiente hemorragia, que no podrá ser controlada sino pasando puntos profundos más o menos ciegos.

Entre lo que nos ha sido posible consultar, encontramos que en 1943 aparece el primer informe de McAusiand, de California, sobre el tratamiento de las várices en la embarazada con sustancias estrogénicas. Se funda McAusland en la hipótesis de que una hormona ovárica o placentaria del tipo de la progesterona sería la que relaja los músculos lisos dei tracto intestinal, ureteres, vías biliares y por consiguiente las venas, ocasionando así las dilataciones varicosas. Esta hormona podría ser contrarrestada con estrógenos, que en la embarazada pueden ser administrados sin riesgo por tiempo prolongado y a dosis altas. No hay en la comunicación referida estadísticas de casos tratados ni plan de tratamiento, sino apenas la sugerencia del sistema.

Posteriormente se ha empleado el procedimiento en bastante escala, habiéndose probado los distintos tipos de estrógenos como el estradiol, estrona, dietilestilbestrol y etinyi estradiol. De acuerdo con McPheeters, de Minneapolis, el que mejores resultados daría sería el etinyl estradiol por vía oral a dosis de cuatro tabletas cilarias de 0.05 mgmos. El tratamiento debe comenzarse tan pron- 
to se ve la paciente por primera vez, y continuarse sin interrupción hasta el séptimo mes. Cerca de un $50 \%$ de ias pacientes de McPheeters tuvieron cesación completa de los síntomas, encontrándose en los otros casos grados variables de mejoría.

De esta revisión podemos concluir algunas nociones prácticas:

a) La mayoría de las várices de las embarazadas son asintomáticas, y fuera de algunas medidas de orden higiénico, no requieren una terapéutica activa y regresan después del parto.

b) Hay casos en que la complicación varicosa del embarazo llega a constituir una verdadera enfermedad, y en estos casos ei obstetra debe tratar de aliviar los síntomas y prevenir las complicaciones.

c) Está demostrado que las várices de las embarazadas pueden ser tratadas médica o quirúrgicamente, sin que esto ocasione complicaciones generales $u$ obstétricas.

d) Ninguno de los tres sistemas fundamentales de tratamiento presenta estadísticas completamente satisfactorias, y cada uno de ellos es considerado como el mejor por sus defensores. Por los datos encontrados, podríamos considerar que todos son iguaimente buenos y que el resultado dependería más que todo de la experiencia de cada uno en determinado método y del análisis cuidadoso de cada caso.

e) En los casos que realmente requieren un tratamiento activo y en que las várices de la embarazada constituyen una enfermedad de cuidado, quizás podrían obtenerse resultados mejores con la aplicación combinada de dos sistemas. Pensamos que la asociación de una cirugía moderada, haciendo ligaduras en los sitios que se consideren claves y administrando conjuntamente estrógenos en dosis adecuadas y por tiempo suficiente, podría ilevar a resultados bastante halagadores.

f) En fin, hemos querido más que todo llamar la atención de los obstetras sobre este punto de la patología que se ha mirado siempre con tanta indiferencia y que en muchas ocasiones constituye un factor fundamental para el bienestar de la grávida. 